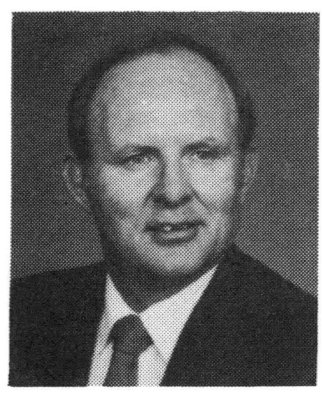

Editorial

Jack D. Gaskill, Editor

Are "Idea-Type" Papers a Good Idea? Reader Response
OPTICAL ENGINEERING EDITORIAL SCHEDULE

April 1988

Industrial Applications of Optical

Signal Processing I

Bahram Javidi

Michigan State University

Electrical Engineering Department

East Lansing, MI 48824-1226

517/353-6329

May 1988

Industrial Applications of Optical

Signal Processing II

Bahram Javidi

(See April 1988)

June 1988

Contributed papers on optical engineering

July 1988

Neural Networks

William J. Miceli

Office of Naval Research

495 Summer Street

Boston, MA 02210-2105

$617 / 451-4484$

\section{August 1988}

Photomechanics II

Fu-Pen Chiang

SUNY/Stony Brook

Lab. for Experimental Mechanics Research

Stony Brook, NY 11794

516/632-8311

\section{September 1988}

Multiple-Aperture Optical Systems

Janet S. Fender

U.S. Air Force Weapons Lab.

Optical Phased Array Branch

Kirtland AFB, NM 87117-6008

505/844-9831

\section{October 1988}

\section{Computer-Aided Optical Design}

Carmina Londoño

Polaroid Corporation

38 Henry Street

Cambridge, MA 02139

$617 / 577-4115$ 